\title{
Smart Garbage Monitoring System using Internet of Things (IOT)
}

\author{
Prof. Dr. Sandeep M. Chaware ${ }^{1}$, Shriram Dighe ${ }^{2}$, Akshay Joshi ${ }^{3}$, Namrata Bajare ${ }^{4}$, Rohini Korke ${ }^{5}$ \\ Faculty, Computer Engineering Dept, TSSM'S BSCOER, Narhe, Pune, India ${ }^{1}$ \\ Student, Computer Dept, BSCOER, Pune, India ${ }^{2,3,4,5}$
}

\begin{abstract}
The Internet of Things (IoT) shall be able to incorporate transparently and seamlessly a large number of different and heterogeneous end systems, while providing open access to selected subsets of data for the development of a plethora of digital services. Building a general architecture for the IoT is hence a very complex task, mainly because of the extremely large variety of devices, link layer technologies, and services that may be involved in such a system. One of the main concerns with our environment has been solid waste management which in addition to disturbing the balance of the environment also has adverse effects on the health of the society. The detection, monitoring and management of wastes is one of the primary problems of the present era. The traditional way of manually monitoring the wastes in waste bins is a complex, cumbersome process and utilizes more human effort, time and cost which is not compatible with the present day technologies in any way. This an advanced method in which waste management is automated. This project IoT Garbage Monitoring system is a very innovative system which will help to keep the cities clean. This system monitors the garbage bins and informs about the level of garbage collected in the garbage bins via a web page. This web page also send all information to garbage collection vehicles.
\end{abstract}

Keywords: Wi-Fi modem, Arduino microcontroller, Application Resource Manager (ARM), Solids Waste management.

\section{INTRODUCTION}

Garbage Monitoring System: - Garbage may consists of the unwanted material left over from City, Public area, Society, College, home etc. This project is related to the "Smart City" and based on "Internet of Things" (IOT). So for smart lifestyle, cleanliness is needed, and cleanliness is begins with Garbage Bin.

This project will helps to eradicate or minimize the garbage disposal problem. The Internet of Things (IoT) is a recent communication paradigm that envisions near future, in which the objects of everyday life will be equipped with microcontrollers, transceivers for digital communication, and suitable protocol stacks that will make them able to communicate with one another and with the users, becoming an integral part of the Internet [1].

This project IOT Garbage Monitoring system is a very innovative system which will help to keep the cities clean. This system monitors the garbage bins and informs about the level of garbage collected in the garbage bins via a web page. For this the system uses ultrasonic sensors placed over the bins to detect the garbage level and compare it with the garbage bins depth. The system makes use of Arduino family microcontroller, LCD screen, Wi-Fi modem for sending data and a buzzer. The system is powered by a $12 \mathrm{~V}$ transformer. The LCD screen is used to display the status of the level of garbage collected in the bins.
Whereas a web page is built to show the status to the user monitoring it. The web page gives a graphical view of the garbage bins and highlights the garbage collected in colour in order to show the level of garbage collected. The LCD screen shows the status of the garbage level. The system puts on the buzzer when the level of garbage collected crosses the set limit. Thus this system helps to keep the city clean by informing about the garbage levels of the bins by providing graphical image of the bins via a web page.

\section{LITERATURE SURVEY}

Some of the following garbage typePackaging waste, Agricultural waste, Inorganic waste, Liquid waste etc.

In solid waste bin monitoring system garbage bin set the public place then Camera set for garbage bin location. The camera captured image for garbage bin. Radio Frequency Identification (RFID), GPS and GIS send image for work station. The RFID reader and camera are mounted in the truck, when truck come closer to the bin RFID reader communicated RFID tag. \& send all information. The System are use controlling Hut. This Controlling Hut are SMS Technology. The GPS and GPRS mapping server to analysing data of various location. The control station compiled all the information and stored in the system database. The bin status and waste truck was monitored. [1] 


\section{International Journal of Innovative Research in Electrical, Electronics, Instrumentation and Control Engineering ISO 3297:2007 Certified}

Vol. 5, Issue 1, January 2017

In waste bin monitoring system using zig bee and Global mobile communication system (GSM).The sensors are place in the common garbage bins placed at the public place when the garbage reaches the level of the sensors. Then that indicated will give in indication to the driver by ARM7 they sending SMS using GSM technology. The technology use by Zig bee, Global mobile system (GSM), ARM 7 Controller.The range of communication of the zig bee is almost 50 meter. They use for range GSM Module, analysing the image we get an idea about level of garbage.The zig bee and GSM system wold be able to monitor the solid waste collection process. This technique overcome some disadvantages which are use of minimum route, low cost, fuel use, clean environment. [2]

The waste management is built around several element. Waste item, domestic bin, trash bags, collective containers and collecting vehicles. The waste flow start from the waste item and the domestic bin to end in the collecting vehicles. Use the waste identification for sorting process. Base on RFID technology new trash bag is added in a collective container. The technology use Radio Frequency Identification (RFID), Smart vehicular and Trash Bag.They only identify RFID tags garbage bins, Low data speed, high cost.The zig bee and GSM system wold be able to monitor the solid waste collection process. This technique overcome some disadvantages which are use of minimum route, low cost, fuel use, clean environment. [3] A single directional cylinder is suspended next to the lid of dustbin. The piston is free to move up and down vertically inside the dustbin to a certain level. A plate is attached to the cylinder for compressing the garbage. The shape of this plate depends upon the shape of the dustbin. The compressing plate consists of a side hole through which the leaf switch is suspended upside down. Technology use Piston, Switch, microcontroller, the single directional cylinder, smart dustbin. Only use for smart dustbins, they are not provide garbage collection.Smart Dustbins can prevent the accumulation of the garbage along the roadside to a great extent thereby controlling the widespread of many diseases. It can prevent pollution and also prevent the consumption of the spread out garbage by the street animals. [4]

A laser diode is a $\mathrm{p}-\mathrm{n}$ junction diode which produces a narrow beam of light that is intense, focused and coherent. In a LASER diode a mirrored resonant chamber is used to reinforce the light waves so that the light emitted by the device is at a single frequency and of the same phase. A photo detector is a device that converts light signals into electrical signals, which can be amplified and processed. Technology use Dustbins, LASER Diode, Photo Detector Diode, Road Side Units (RSU), and Garbage Collecting Vehicle (GCV).Only support for simulation of Transmission Control Protocol (TCP), routing and multicast protocols over wired.The dynamic routing of GCV compared with static solution is much more efficient and will be much effective when more than one dustbin fills up at the same time. The initial planned route is saved so that when real-time data is received only portion of the planned path may be changed. [5]

For the garbage detection, weight sensor can be used. It gives the weight of the garbage in the dustbin. But it doesn't provide any information about the level of the garbage in the dustbin. Hence author used Infrared (IR) sensor for garbage detection. IR sensor radiates light which is invisible to the human eye because it is at infrared wavelengths, but it can be detected by electronic devices.IR transmitter consists of LED which send the IR beam. Technology use Infra-red sensor (IR), Microcontroller, Global System for Mobile (GSM), graphical user interface (GUI).Infrared sensor (IR), Global System for Mobile (GSM).They only use GSM network. Power and internet supply continue on.Smart garbage management system using IR sensor, microcontroller and GSM module. This system assures the cleaning of dustbins soon when the garbage level reaches its maximum. [6]

Arduino is best described as a single-board computer that has deliberately been designed to be used by people who are not experts in electronics, en-Gingering, or programming. It is inexpensive, cross-platform (the Arduino software runs on Windows, Mac OS X, and Linux), and easy to program. Both Arduino hardware and software are open source and extensible.Arduino is also powerful: despite its compact size, it has about as much computing muscle as one of the original navigation computers from the Apollo Programmers, designers, do-ityourselves, and artists around the world take advantage of Arduino's power and simplicity to create all sorts of innovative devices, including interactive sensors, artwork, and toys. [7]

\section{SYSTEM ARCHITECTURE}

The IOT Garbage Monitoring system is a very innovative system which will help to keep the cities clean. This system monitors the garbage bins and informs about the level of garbage collected in the garbage bins via a web page. For this the system uses ultrasonic sensors placed over the bins to detect the garbage level and compare it with the garbage bins depth. The system makes use of Arduino family microcontroller, LCD screen, Wi-Fi modem for sending data and a buzzer. The system is powered by a $12 \mathrm{~V}$ transformer. The LCD screen is used to display the status of the level of garbage collected in the bins.

Whereas a web page is built to show the status to the user monitoring it. The web page gives a graphical view of the garbage bins and highlights the garbage collected in colour in order to show the level of garbage collected. The LCD screen shows the status of the garbage level. The system puts on the buzzer when the level of garbage collected crosses the set limit. Thus this system helps to keep the 
city clean by informing about the garbage levels of the bins by providing graphical image of the bins via a web page.

The ESP8266 Wi-Fi Module is a self-contained SOC with integrated TCP/IP protocol stack that can give any microcontroller access to your Wi-Fi network. The
ESP8266 is capable of either hosting an application or offloading all Wi-Fi networking functions from another application processor. Each ESP8266 module comes preprogramed with an AT command set firmware. The ESP8266 module is an extremely cost effective board with a huge, and ever growing, community.

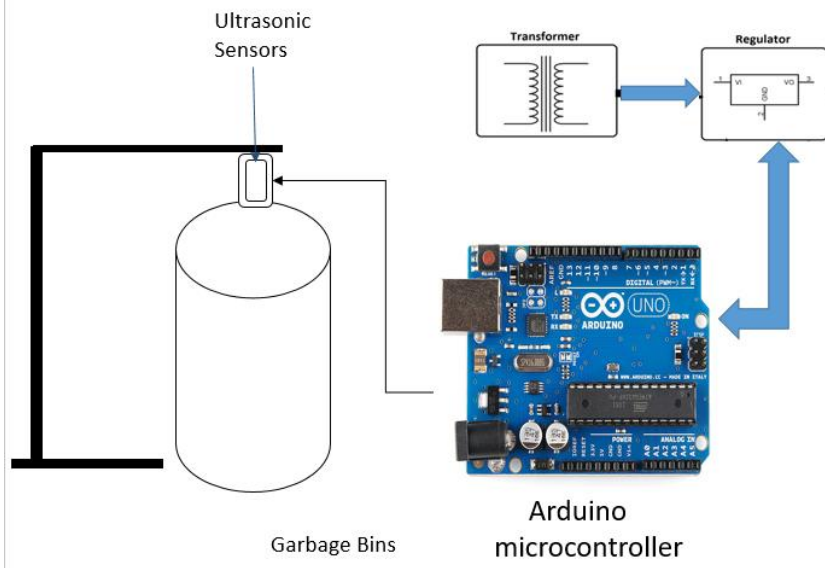

Figure: System Architecture Diagram

\section{HARDWARE USED:}

Microcontroller: It get information from sensor and process on it. It compares the received data with the threshold level set and accordingly output is generated. The LPC2131/32/34//38 microcontrollers are based on a 16/32-bit ARM7TDMI-S CPU with real-time emulation and embedded trace support, that combine the microcontroller with $32 \mathrm{kB}, 64 \mathrm{kB}, 128 \mathrm{kB}, 256 \mathrm{kB}$ and $512 \mathrm{kB}$ of embedded high-speed flash memory.A128-bit wide memory interface and unique accelerator architecture enable 32-bit code execution at maximum clock rate.

Power Supply: We use 12v power supply in our project. It is mainly used to provide DC voltage to the components on board. $3.3 \mathrm{~V}$ for $\mathrm{lpc} 2138$ and $4.2 \mathrm{v}$ for $\mathrm{Wi}-\mathrm{Fi}$ module is apply from power supply. $5 \mathrm{~V}$ is required for relay applied from power supply.

WI-FI Modem: This module has a powerful enough onboard processing and storage capability that allows it to be integrated with the sensors and other application specific devices through its GPIOs with minimal development upfront and minimal loading during runtime. Its high degree of on-chip integration allows for minimal external circuitry, including the front-end module, is designed to occupy minimal PCB area. The ESP8266 supports APSD for VoIP applications and Bluetooth co-existence interfaces, it contains a self-calibrated RF allowing it to work under all operating conditions, and requires no external RF parts.
There is an almost limitless fountain of information available for the ESP8266, all of which has been provided by amazing community support. In the Documents section below you will find many resources to aid you in using the ESP8266, even instructions on how to transforming this module into an IoT (Internet of Things) solution! Note: The ESP8266 Module is not capable of 5-3V logic shifting and will require an external Logic Level Converter. Please do not power it directly from your $5 \mathrm{~V} \mathrm{dev}$. board.

Ultrasonic Sensor: The Ultrasonic Sensor sends out a high-frequency sound pulse and then times how long it takes for the echo of the sound to reflect back. The sensor has 2 openings on its front. One opening transmits ultrasonic waves, (like a tiny speaker), the other receives them, (like a tiny microphone). The speed of sound is approximately 341 meters (1100 feet) per second in air. The ultrasonic sensor uses this information along with the time difference between sending and receiving the sound pulse to determine the distance to an object.

GSM Module: It is used to send message to the garbage depot if the Garbage Can exceeds the set threshold level. With the help of GSM module interfaced, we can send short text messages to the required municipal office. GSM module is provided by sim uses the mobile service provider and send sms to the respective authorities as per programmed. It operates at either the $900 \mathrm{MHz}$ or 1800 $\mathrm{MHz}$ frequency band. 


\section{FLOWCHART:}

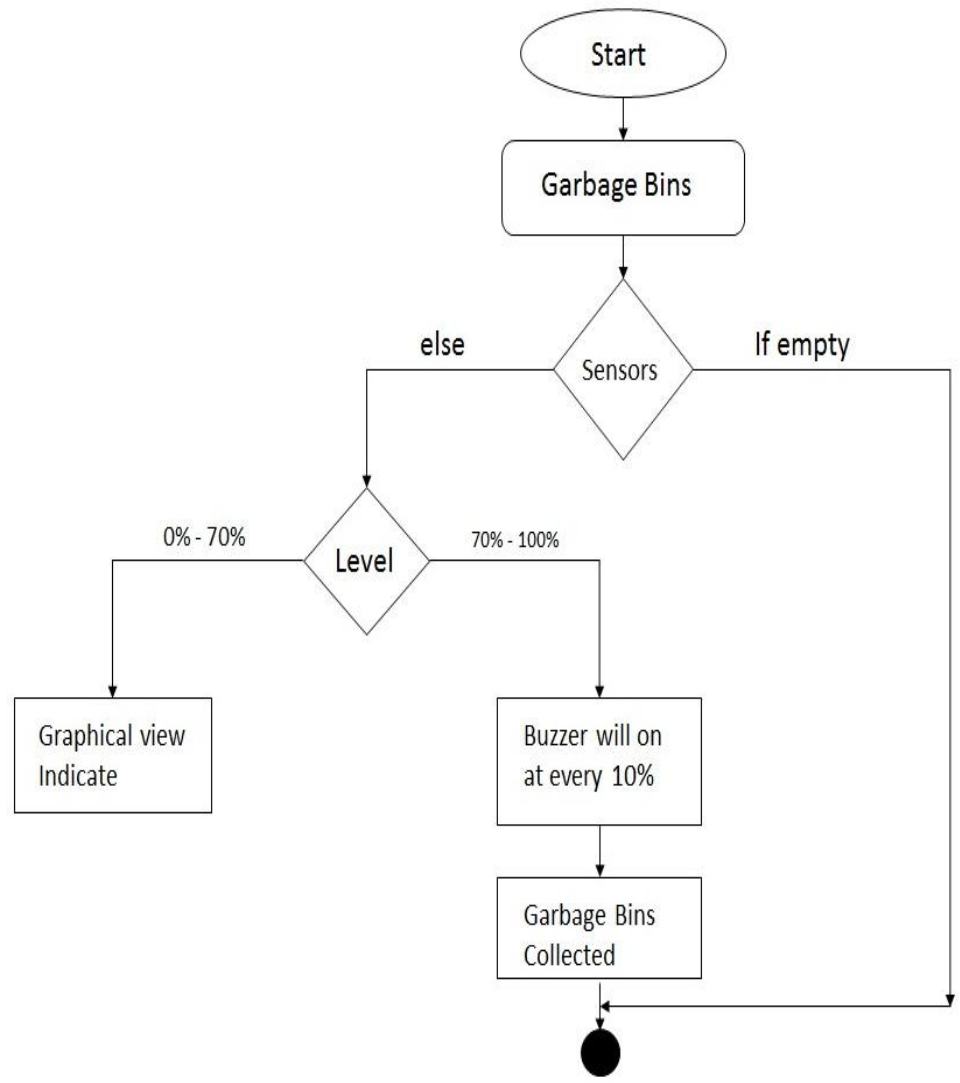

\section{ADVANTAGES:}

Monitors the garbage bins and informs about the level of garbage collected in the garbage bins.

To keep our Environment clean \& green.

The cost $\&$ effort are less in this system.

\section{APPLICATIONS:}

This project can also be used in the" SMART CITY", This project is also helpful in the government project of "SWACHH BHARAT ABHIYAN".

\section{CONCLUSION}

In this project, an integrated system of Wi-Fi modem, IoT, GSM, Ultrasonic Sensor is introduced for efficient and economic garbage collection. The developed system provides improved database for garbage collection time and waste amount at each location. We analysed the solutions currently available for the implementation of IoT. By implementing this project we will avoid over flowing of garbage from the container in residential area which is previously either loaded manually or with the help of loaders in traditional trucks. It can automatically monitor the garbage level \& send the information to collection truck. The technologies which are used in the proposed system are good enough to ensure the practical and perfect for solid garbage collection process monitoring and management for green environment.

\section{REFERENCES}

[1] Prof. R.M.Sahu, Akshay Godase, Pramod Shinde, Reshma Shinde, "Garbage and Street Light Monitoring System Using Internet of Things" INTERNATIONAL JOURNAL OF INNOVATIVE RESEARCH IN ELECTRICAL, ELECTRONICS, INSTRUMENTATION AND CONTROL ENGINEERING, ISSN (Online) 2321 - 2004, Vol. 4, Issue 4, April 2016.

[2] Kanchan Mahajan, Prof.J.S.Chitode, "Waste Bin Monitoring System Using Integrated Technologies", International Journal of Innovative Research in Science, Engineering and Technology (An ISO 3297: 2007 Certified Organization) Vol. 3, Issue 7, July 2014.

[3] Md. Shafiqul Islam, M.A. Hannan, Maher Arebey , Hasan Basri , “An Overview For Solid Waste Bin Monitoring System", Journal of Applied Sciences Research, ISSN 181-544X, vol.5,lssue4, February 2012.

[4] Twinkle sinha, k.mugesh Kumar, p.saisharan, "SMART DUSTBIN", International Journal of Industrial Electronics and Electrical Engineering, ISSN: 2347-6982 Volume-3, Issue-5, May2015

[5] Richu Sam Alex, R Narciss Starbell, "Energy Efficient Intelligent Street Lighting System Using ZIGBEE and Sensors", International Journal of Engineering and Advanced Technology (IJEAT) ISSN: 2249 - 8958, Volume-3, Issue-4, April 2014.

[6] Narendra Kumar G., Chandrika Swami, and K. N. Nagadarshini, "Efficient Garbage Disposal Management in Metropolitan", Cities Using VANETs Journal of Clean Energy Technologies, Vol. 2, No. 3, July 2014.

[7] Emily Gertz, Patrick Di Justo,'Environmental Monitoringwith Arduino"Copyright (C) 2012 Emily Gertz and Patrick Di Justo. All rights reserved. Printed in the United States of America,ISBN: 9781-449-31056-1, January 20, 2012. 